\title{
Revisão Sistemática
}

\section{Medo de cair e o risco de queda: revisão sistemática e metanálise}

\author{
Fear of falling and risk of falling: a systematic review and meta-analysis \\ Miedo de caer y riesgo de caída: revisión sistemática y metanálisis
}

Silvana Barbosa Pena ${ }^{1}$

Heloísa Cristina Quatrini Carvalho Passos Guimarães ${ }^{2}$

Juliana Lima Lopes ${ }^{3}$

Lidia Santiago Guandalini ${ }^{3}$

Mônica Taminato ${ }^{3}$

Dulce Aparecida Barbosa ${ }^{3}$

Alba Lúcia Bottura Leite de Barros

\section{Descritores}

Idoso; Acidente por quedas; Medo; Fatores de risco

\section{Keywords}

Aged; Accidental falls; Fear; Risk factors

\section{Descriptores}

Anciano; Accidentes por caídas; Miedo; Factores de riesgo

\section{Submetido}

17 de Setemebro de 2018

\section{Aceito}

20 de Maio de 2019

\section{Resumo}

Objetivo: Verificar se o medo de cair é fator de risco em pessoas idosas que vivem na comunidade.

Métodos: Foi realizada uma revisão sistemática com metanálise baseada na Preferred Reporting Items for Systematic Reviews and MetaAnalyses Utilizou-se a estratégia de busca PECOS: Paciente - pessoa idosa com 60 anos, ou mais, que vive em comunidade com história pregressa de quedas: Exposição- medo de cair, Comparação - grupo sem medo de cair, o "Outcome" - o desfecho queda e 0 "Studies"- foram incluídos os estudos observacionais comparativos. As buscas foram realizadas em maio de 2018 nos seguintes bancos de dados eletrônicos CINAHL, Medline, Cochrane, Embase, Lilacs, PSycINFO e PEDro, por meio dos seguinte descritores: "aged", "elderly", older adults, fear, fear of falling, accidental falls, fall, fallls. Foram também realizadas buscas de referências cruzadas e literatura cinzenta. Dois revisores realizaram a identificação, seleção, elegibilidade e inclusão dos estudos de maneira independente. A qualidade metodológica dos estudos foram efetuada pela aplicação do instrumento STROBE. Para a metanálise, utilizou-se o Programa ReviewMananger $5.3^{\circledR}$

Resultados: De 4.891 publicacões, cinco estudos possibilitaram a metanálise com 3.112 idosos. Evidenciou-se uma chance de queda de 12,15 vezes maior para o grupo de idosos com medo de cair.

Conclusão: 0 medo de cair foi identificado como fator de risco de queda na população idosa que vive na comunidade e que possui história pregressa de queda, torna-se necessário a investigação pelos profissionais da ara da saúde afim de estabelecer medidas preventivas.

\section{Abstract}

Objective: to verify if fear of falling is a risk factor in older people living in the community.

Methods: A systematic review was conducted with a meta-analysis based on the Preferred Reporting Items for Systematic Reviews and MetaAnalyses. The PECOS search strategy was used: Patient - elderly person aged 60 years or above, living in a community with a previous history of falls; Exposure - Fear of falling, Comparison - group without fear of falling, "Outcome" - fall outcome and "Studies" - included comparative observational studies. The searches were conducted in May 2018 in the following electronic databases CINAHL, Medline, Cochrane, Embase, Lilacs, PsycINFO and PEDro, through the following descriptors: "aged", "elderly", older adults, fear, fear of falling, accidental falls, fall, falls. Crossreferences and gray literature were also searched. Two reviewers independently performed the identification, selection, eligibility and inclusion of the studies. The methodological quality of the studies was carried out by applying the STROBE tool. For the meta-analysis, the Review Mananger 5.3 Program was used ${ }^{\circledR}$

Results: Of 4,891 publications, five studies enabled a meta-analysis with 3,112 elderly. There was a chance of a fall of 12.15 times higher for the group of elderly people with fear of falling.

Conclusion: Fear of falling was identified as a risk factor for falling in the elderly population that lives in the community and that has a history of falling, it is necessary the investigation by health professionals in order to establish preventive measures.

\section{Resumen}

Objetivo: verificar si el miedo de caer es factor de riesgo en personas mayores que viven en la comunidad.

Métodos: se realizó una revisión sistemática con metanálisis basada en la Preferred Reporting Items for Systematic Reviews and MetaAnalyses. Se utilizó la estrategia de búsqueda PECOS: Paciente (ancianos con 60 años o más, que viven en comunidad con historial anterior de caídas); Exposición (miedo de caer); Comparación (grupo sin miedo de caer): "Outcome" (el desenlace de la caída) y "Studies" (fueron incluidos los estudios observacionales comparativos). Las búsquedas fueron realizadas en mayo de 2018 en los siguientes bancos de datos electrónicos CINAHL, Medline, Cochrane, Embase, Lilacs, PSycINFO y PEDro, por medio de los siguientes descriptores: "aged", "elderly", older adults, fear, fear of falling, accidental falls, fall, fallls. También se realizaron búsquedas de referencias cruzadas y literatura gris. Dos revisores llevaron a cabo la identificación, selección, elegibilidad e inclusión de los estudios de manera independiente. La calidad metodológica de los estudios fue efectuada mediante la aplicación del instrumento STROBE. Para el metanálisis, se utilizó el programa ReviewMananger $5.3^{\circledR}$.

Resultados: de 4.891 publicaciones, 5 estudios posibilitaron el metanálisis con 3.112 ancianos. Se observó una posibilidad de caída 12,15 veces mayor en el grupo de ancianos con miedo a caer.

Conclusión: el miedo a caer fue identificado como factor de riesgo de caída en personas mayores que viven en la comunidad y que poseen historial anterior de caída. Resulta necesaria la investigación por parte de profesionales del área de la salud a fin de establecer medidas preventivas.

\section{Como citar:}

Pena SB, Guimarães HC, Lopes JL, Guandalini LS, Taminato M, Barbosa DA, et al. Medo de cair e o risco de queda: revisão sistemática e metanálise. Acta Paul Enferm. 32(4):456-63. 


\section{Introdução}

As projeçóes sobre o envelhecimento populacional no mundo foram apresentadas no Relatório Mundial sobre Envelhecimento e Saúde desenvolvido pela Organização Mundial de Saúde (OMS) em 2015, e aponta que o número de pessoas com mais de 60 anos duplicará até o ano de 2050 no mundo e, no Brasil, ela quase triplicará. Os idosos correspondem a $12,5 \%$ do total da população brasileira e até a metade do século, poderão atingir o percentual de $30 \%$, passando o Brasil a ser considerado como uma "Nação Envelhecida". Este termo é atribuído, pela OMS, aos países que são compostos por mais de $14 \%$ de pessoas idosas. Outro dado que é destacado pelo Relatório Mundial sobre Envelhecimento e Saúde é que, dentre os problemas de saúde da população idosa, a queda é um deles. ${ }^{(1)}$

Dentre os fatores de risco de quedas na população idosa, o medo de cair tem chamado a atenção, tanto nos achados da literatura científica nacional e internacional bem como na prática clínica do enfermeiro, e de outros profissionais da área da saúde. ${ }^{(2,3)}$

O medo de cair (ptophobia) foi descrito, como consequências psicológicas e comportamentais de uma queda sofrida por uma pessoa idosa. Em um estudo evidenciado na literatura, pesquisadores acompanharam 36 pacientes admitidos em um hospital devido à queda e observaram que, após quatro meses do evento, apresentaram um conjunto de sinais e sintomas que compóem a "síndrome de pós-queda", em inglês "post-fall syndrome", sendo o medo de cair o sintoma mais prevalente. ${ }^{(4)}$

Discussóes a respeito do conceito de medo de cair e o seu reconhecimento enquanto fobia específica, bem como a natureza do medo de cair, tem remetido a várias reflexóes, principalmente quanto aos constructos associados à sua ocorrência. Assim, o fato de o idoso que não vivenciou nenhuma queda desenvolver medo de cair tem fortalecido a importância da inter-relação de outros fatores associados a esse medo, podendo ser considerado como um fenômeno multifatorial, náo reduzido a um medo de voltar a cair e/ou sofrer as consequências da queda. ${ }^{(5)}$

Outro conceito sobre o medo de cair é constituído por três diferentes elementos: o cognitivo, o fisiológico e o comportamental. Esses elementos facilitam o entendimento do medo de cair e uma melhor estimativa do risco de quedas. $\mathrm{O}$ mesmo modelo sugere que o medo de cair sobrevém da percepção que a pessoa idosa possui em sua capacidade manter o equilíbrio e lidar com as quedas, juntamente com outros fatores, como a ocorrência de quedas e as crenças que tem acerca desses eventos, mas não de um modo causal, isto é, o modelo assume que o medo de cair é fortemente associado à ocorrência de quedas, não sendo um resultado automático das mesmas, já que as pessoas com história pregressa de queda e as que nunca caíram poderão desenvolver este medo. ${ }^{(5)}$

O Ministério da Saúde do Brasil define o medo de cair como: "um sentimento de grande inquietação diante a noção de um perigo real, aparente ou imaginário de quedas, ou seja, pode estar presente mesmo no idoso que nunca caiu". ${ }^{(6)}$

Encontram-se na literatura estudos que utilizaram ferramentas para identificar o medo de cair desde a uma simples pergunta "Você tem medo de cair? Que vêm muitas vezes acompanhada de perguntas que avaliam a intensidade ou a frequência do medo de cair, até a aplicação de escala Falls Efficacy Scale (FES) Escala de Autoeficácia de Queda contendo atividades da vida diária onde a mesma estabelece uma correlação da queda com a autoeficácia, apoiados na Teoria Social Cognitiva, definida como "baixa autoeficácia percebida para evitar quedas durante atividades da vida diária”. ${ }^{(7)}$ Ainda no que se diz respeito a esta escala, desenvolveu-se a versão curta: Short FES-I com sete perguntas e com um escore variando entre 7 a 28. Se comparado ao FES-I versão longa, demonstrou menor tempo de resposta, o que foi evidenciado em estudos por meio da avaliação de propriedades psicométricas, apresentando alta confiabilidade interna com alpha de Cronbach de 0, 92. ${ }^{(8)}$

Identifica-se, na Classificação Internacional de diagnósticos de enfermagem da NANDA-I a presença do diagnóstico Risco de quedas que é definido como a "suscetibilidade aumentada a quedas que pode causar dano físico e comprometer a saúde", no entanto observa-se que, dentre os fatores de risco identificados deste diagnóstico não é identificado o medo de cair como um fator de risco. ${ }^{(9)}$ 
O medo de cair na pessoa idosa pode desencadear restrição de atividades, limitação da capacidade física e isolamento social, desse modo identificar as evidências existentes na literatura sobre o medo de cair como um fator de risco para quedas instrumentaliza os enfermeiros e a equipe multiprofissional para seu reconhecimento e implementação de intervençóes com o objetivo de prevenir quedas. ${ }^{(10,11)}$ Assim, o presente estudo objetivou verificar se o medo de cair é fator de risco em pessoas idosas que vivem na comunidade.

\section{Métodos}

Foi realizada uma revisão sistemática com metanálise, baseada nas diretrizes metodológicas: Elaboração de revisão sistemáticas e metanálise de estudos observacionais comparativos sobre fatores de risco e prognósticos, ${ }^{(12)}$ guiada pela seguinte pergunta de pesquisa "Existem evidências científicas na literatura sobre o medo de cair como fator de risco ou protetor para quedas na pessoa idosa com história pregressa de quedas?", sustentada na estratégia PECOS: Paciente - pessoa idosa com 60 anos, ou mais, que vive em comunidade com história pregressa de quedas; Exposição- medo de cair, Comparação -grupo sem medo de cair, o "Outcome" - o desfecho queda e o "Studies"- foram incluídos os estudos observacionais comparativos.

\section{Critérios de exclusão}

Foram excluídos os estudos com idosos que sofreram queda e que apresentaram comorbidades, tais como Parkinson, Alzheimer, fraturas de fêmur, idosos frágeis, claudicação, câncer, entre outras afim de diminuir variáveis confundidoras.

\section{Busca e identificação dos artigos}

As bases eletrônicas pesquisadas foram CINAHL, Medline/PubMed, Embase, SPORTDISCUS, Lilacs, PsycINFO e PEDro. A busca na literatura foi realizada em maio de 2018, no entanto não delimitou-se ano de publicação e idioma, conforme as diretrizes metodológicas de revisão sistemática e metanálise. ${ }^{(12)}$ Outras fontes de busca foram inves- tigadas por meio da literatura cinzenta e de busca manual: referência cruzada dos estudos incluídos. Para a busca, foram utilizados descritores controlados de acordo com os Descritores em Ciências da Saúde (DeSC), PubMed, Medical Subject Headings (MeSH) e utilizados os operadores boleanos "and" e "or". Utilizamos os seguintes termos de busca no PubMed e adaptamos a estratégia para as outras bases de dados:"Aged"[Mesh] OR aged OR elderly OR "Aged, 80 and over"[Mesh] OR" Oldest Old" OR Nonagenarian* OR Octogenarian* OR Centenarian* $O R$ "older adult" $O R$ "older adults" $O R$ older $O R$ senior "Fear" [Mesh] OR fear OR fears OR "Phobic Disorders"[Mesh]OR "Disorder, Phobic" OR "Phobic Disorder" OR "Phobic Neuroses" OR "Neuroses, Phobic" OR Phobias OR Phobia OR "Panic"[Mesh] $O R$ panic $O R$ panics $O R$ "fear of falling" "Accidental Falls"[Mesh] OR Falls OR fall OR Falling OR "Falls Accidental" OR "Accidental Fall" OR "Fall, Accidental"\#1 AND \#2 AND \#3. Os estudos foram selecionados utilizando o Preferred Reporting Items for Systematic Reviews and Meta-Analyse (PRISMA) por meio da identificação, seleção e avaliação da elegibilidade. ${ }^{(16)}$ Após a seleção do estudos, foram excluídos artigos duplicados por meio do software (EndNoteBasic ${ }^{\oplus}$, Thomson Reuters, USA).

\section{Seleção dos estudos}

A seleção dos estudos foi realizada de maneira independente por dois revisores, por meio da plataforma de seleção Rayyan. Inicialmente os artigos foram selecionados após a leitura do título e resumo e os que atenderam os critérios de elegibilidade e que tiveram consenso entre os dois revisores, foram lidos na íntegra para inclusão ou exclusão na revisão. As discordâncias na fase da leitura na íntegra foram resolvidas por consenso por um terceiro revisor. ${ }^{(12)}$

\section{Sumarização dos dados}

Os estudos incluídos tiveram seus dados sumarizados por dois avaliadores, utilizando para isso o formulário de extração de dados modificado do instrumento proposto pela Diretrizes Metodológicas: elaboração de revisão sistemáticas e metanálise de estudos observacionais comparativos sobre fatores de risco e prognósticos, identificando autor e ano 
de publicação, país, tipo de estudo, idade, tamanho da amostra, ferramenta para identificar o medo de cair e qualidade metodológica do estudo. ${ }^{(12)}$

\section{Avaliação da qualidade metodológica}

A avaliação da qualidade metodológica dos estudos incluídos foi efetuada pela aplicação do instrumento STROBE (Strengthening the Reporting of Observational Studies in Epidemiology). ${ }^{(12)}$

\section{Síntese dos resultados}

Para a metanálise, utilizou-se o Programa ReviewMananger $5.3^{\circ}$. As medidas de associação de risco de queda foram analisadas por meio do cálculo de $\operatorname{OddsRatio(OR)}$ com Intervalo de Confiança de $95 \%$ e nível de significância de 5\%. Quando possível, as medidas de associação dos estudos individuais foram combinadas em uma metanálise com gráfico de floresta (forestplot) com modelo estatístico Mantel-Haenszel para combinar diferentes $O R$. $\mathrm{O}$ modelo de análise de efeito foi determinado de acordo com a heterogeneidade dos resultados avaliada pela estatística $\mathrm{I}^{2}$. A presença de heterogeneidade estatística foi pesquisada pela inspeçáo da apresentação dos gráficos da metanálise e do gráfico do funil ("funnelplot"). Também foi calculado o I-quadrado $\left(\mathrm{I}^{2}\right)$ para pesquisa de heterogeneidade, em que valores de $\mathrm{I}^{2}$ maior ou igual a 50\% são considerados como heterogêneos. Diante de uma heterogeneidade elevada ( $\mathrm{I}^{2}$ maior que $\left.50 \%\right)$, foi utilizado o software Cochrane Review Manager 5.3 $3^{\circ}{ }^{(12)}$

\section{Resultados}

Identificou-se nas bases eletrônicas 4.885 artigos, seis estudos foram identificados por outras fontes, totalizando 4.891 estudos, dos quais 1.392 foram excluídos por serem referências duplicadas. Após a exclusão, 3.499 estudos foram lidos e avaliados pelo título e resumo. Após a leitura do título e resumo, 3.650 artigos não atenderam aos critérios de elegibilidade, sendo que apenas 14 artigos foram lidos na íntegra. Após a leitura na íntegra, foram excluídos sete artigos pelas seguintes razóes: idade menor ou igual a 58 anos, ${ }^{(13)}$ estudo do tipo descritivo ${ }^{(14)}$ idosos em condição específica de saúde ${ }^{(15-17)}$ e falta de clareza dos dados. ${ }^{(18,19)}$ Sete artigos foram entăo, incluídos na revisão. ${ }^{(20-26)} \mathrm{O}$ total dos artigos incluídos que atenderam aos critérios de inclusão foram sete, estes permitiram a análise qualitativa(20-26) Sendo possível a análise quantitativa somente para cinco artigos. ${ }^{(20-22,25,26)} \mathrm{A}$ razão da não inclusão dos dois artigos na avaliaçáo quantitativa se deram devido ao medo de cair ser avaliado por diferentes escalas (Figura 1). ${ }^{(23,24)}$

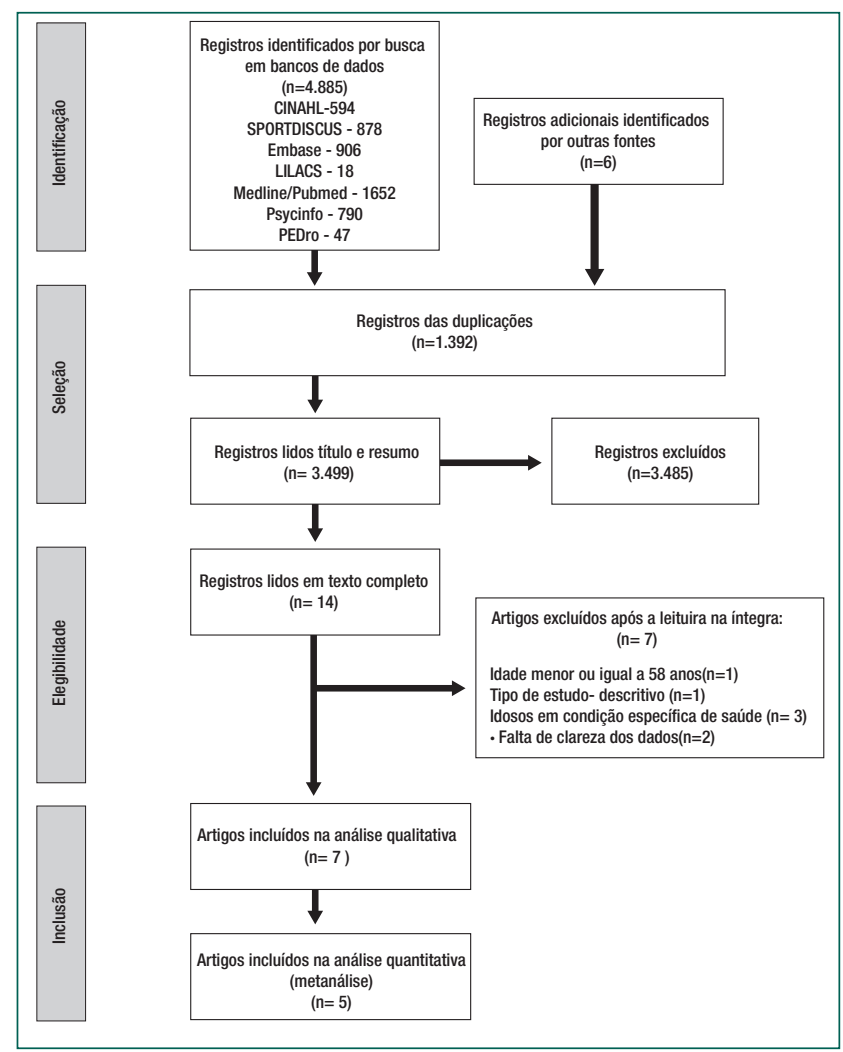

Figura 1. Fluxograma da seleção de evidências baseado nas diretrizes do PRISMA

$\mathrm{O}$ resumo das características gerais dos sete estudos incluídos ${ }^{(25,26)}$ foram quatro transversais, ${ }^{(21-24)}$ três longitudinais prospectivos. ${ }^{(20,25,26)}$ Os sete artigos foram de diferentes países, tais como Espanha, ${ }^{(26)}$ México, ${ }^{(25)}$ Índia, ${ }^{(24)}$ China, ${ }^{(23)}$ Coreia, ${ }^{(22)}$ Canadá ${ }^{(21)}$ e Estados Unidos. ${ }^{(20)}$ Todos os estudos $^{(20-26)}$ foram publicados entre o período de 2005 a 2018, a faixa etária dos idosos foi de 60 anos ou mais, o tamanho da amostra dos estudos variou entre 250 a 9033 idosos que vivem na comunidade. As diferentes ferramentas utilizadas para identificar 
Quadro 1. Descrição das características dos estudos incluídos e avaliação da qualidade metodológica

\begin{tabular}{|c|c|c|c|c|c|c|}
\hline $\begin{array}{l}\text { Autor /Ano de } \\
\text { publicação }\end{array}$ & País & Tipo de estudo & Idade & Tamanho da amostra & $\begin{array}{l}\text { Ferramenta para } \\
\text { Identificar o medo de cair }\end{array}$ & Strobe \\
\hline Dierking MAL, 2016(25) & México & $\begin{array}{l}\text { Estudo Longitudinal } \\
\text { prospectivo }\end{array}$ & $\geq 72$ anos & $\begin{array}{l}1.682 \\
\text { Idosos }\end{array}$ & $\begin{array}{l}\text { Pergunta simples e escala de intensidade de medo de cair. Quão você tem } \\
\text { medo de cair? Escala de Likert: sem medo, algum medo bastante medo } \\
\text { e medo extremo (severo medo de cair).Dicotomizadas em escore: } 0=\text { não } \\
\text { medo e } 1 \text { = para as outras opções. }\end{array}$ & A \\
\hline $\begin{array}{l}\text { Filiatrault J, Desrosiers } \\
\text { J, 2011(21) }\end{array}$ & Canadá & Estudo Transversal & $\geq 65$ anos & $\begin{array}{l}288 \\
\text { Idosos }\end{array}$ & $\begin{array}{l}\text { Pergunta simples e escala de frequência de medo de cair. } \\
\text { Você tem medo de cair? } \\
\text { Categorizada em: nunca, ocasionalmente, frequentemente e muito } \\
\text { frequentemente. } \\
\text { Dicotomizadas em escore: } 0=\text { nunca } 1=\text { para as outras opções. }\end{array}$ & A \\
\hline Kim S, So WY,2013 (22) & Coreia & Estudo Transversal & $>60$ anos & $\begin{array}{l}9.033 \\
\text { Idosos }\end{array}$ & $\begin{array}{l}\text { Pergunta simples e escala de intensidade de medo de cair. } \\
\text { Você tem medo de cair? (cair, escorregar ou cair ao sentar) } \\
\text { Categorizada em: sem medo, algum medo e medo extremo. Dicotomizadas } \\
\text { em escore: } 0=\text { sem medo } 1=\text { com algum medo/medo }\end{array}$ & A \\
\hline Lach H W, 2005(20) & $\begin{array}{l}\text { Estados } \\
\text { Unidos }\end{array}$ & $\begin{array}{l}\text { Estudo longitudinal } \\
\text { prospectivo }\end{array}$ & $\geq 60$ anos & $\begin{array}{l}890 \\
\text { Idosos }\end{array}$ & $\begin{array}{l}\text { Pergunta simples e escala de frequência de medo. } \\
\text { No momento, você tem muito medo, algum medo ou não tem medo de cair } \\
\text { (cair novamente)? } \\
\text { Dicotomizadas em escore: } 0=\text { não medo e } 1 \text { = algum/muito medo. }\end{array}$ & A \\
\hline Lavedán et al., 2018(26) & Espanha & $\begin{array}{l}\text { Estudo longitudinal } \\
\text { prospectivo }\end{array}$ & $\geq 75$ anos & $\begin{array}{c}640 \\
\text { Idosos }\end{array}$ & $\begin{array}{l}\text { Pergunta simples Você tem medo de cair? Com respostas dicotômicas: } \\
\text { sim/não. }\end{array}$ & A \\
\hline Liu J YW, 2014(23)* & China & Estudo Transversal & $\geq 65$ anos & $\begin{array}{c}445 \\
\text { Idosos }\end{array}$ & CFES-I & A \\
\hline Mane et al., 2014(24) $)^{*}$ & Índia & Estudo Transversal & $>60$ anos & $\begin{array}{l}250 \\
\text { Idosos }\end{array}$ & Short FES-I & A \\
\hline
\end{tabular}

*CFES-I - Chinese Fall Efficacy Scale- International; *Short FES-I - Short Fall Efficacy Scale- International; Estudos incluídos na análise qualitativa e excluídos da metanálise

\begin{tabular}{|c|c|c|c|c|c|c|c|c|c|}
\hline Study or Subgroup & \multicolumn{2}{|c|}{ FOF with falls } & \multicolumn{2}{|c|}{ No FOF with falls } & Weight & $\begin{array}{l}\text { Odds Ratio } \\
\text { M-H, Fixed, } 95 \% \mathrm{Cl}\end{array}$ & \multicolumn{2}{|c|}{$\begin{array}{c}\text { Odds Ratio } \\
\text { M-H, Fixed, } 95 \% \mathrm{Cl}\end{array}$} & \\
\hline Dierking L et al. 2016(25) & 470 & 594 & 124 & 594 & $14.9 \%$ & $14.37[10.86,19.01]$ & \multirow{5}{*}{-} & \multirow{5}{*}{${ }^{-}$} & \multirow{5}{*}{ - } \\
\hline Filiatrault J, Desrosiers J, 2011(21) & 40 & 61 & 21 & 61 & $4.2 \%$ & $3.63[1.72,7.66]$ & & & \\
\hline Kim S, So WY. 2013(22) & 1539 & 1604 & 64 & 1604 & $1.5 \%$ & $560.60[394.66,796.31]$ & & & \\
\hline Lach HW, 2005(20) & 84 & 213 & 129 & 213 & $45.0 \%$ & $0.42[0.29,0.63]$ & & & \\
\hline Lavedan A et al. 2018 (26) & 234 & 640 & 94 & 640 & $34.4 \%$ & $3.35[2.55,4.39]$ & & & \\
\hline Total $(95 \% \mathrm{Cl})$ & & 3112 & & 3112 & $100.0 \%$ & $12.15[10.74,13.74]$ & & 1 & \\
\hline Total events & 2367 & & 433 & & & & & & \\
\hline $\begin{array}{l}\text { Heterogeneity: } \mathrm{Chi}^{2}=842.12, \mathrm{df}=4 \\
\text { Test for overall effect: } \mathrm{Z}=39.69(\mathrm{P}\end{array}$ & D.00001); & $=100 \%$ & & & & & 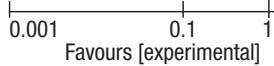 & $\begin{array}{c}10 \\
\text { Favours [control] }\end{array}$ & 1000 \\
\hline
\end{tabular}

Figura 2. Razão de chance de queda entre idosos com e sem medo de cair com história pregressa de queda

o medo de cair foram perguntas simples e direta com respostas dicotômicas e com escala analógica de intensidade e frequência encontradas em cinco estudos, ${ }^{(20-22,25,26)}$ sendo que dois estudos ${ }^{(23,24)}$ aplicaram escalas diferentes, como a Chinese Fall Efficacy Scale-International-CFES-I $I^{(23)}$ e a versão curta Short FES-I. ${ }^{(24)}$ Ao avaliar a qualidade metodológica dos sete estudos incluídos, ${ }^{(20-26)}$ observou-se que todos preencheram mais do que $80 \%$ dos critérios do instrumento STROBE. A sumarização dos dados estão relacionadas no quadro 1.

Cinco estudos, ${ }^{(20-22,25,26)}$ puderam ser combinados em uma metanálise para analisar a razão de chance de queda entre idosos com e sem medo de cair que tiveram história pregressa de queda. Comparando os eventos de queda para os idosos abordados com a pergunta simples e direta com respostas dicotómicas e com escala analógica, obser- vou-se, que o grupo de idosos que responderam que têm medo de cair apresentaram uma razão de chance de queda de 12,15 (IC=10,74-13,74) em relação ao grupo sem medo de cair. A heterogeneidade entre os estudos incluídos foi de $\mathrm{I}^{2}=100 \%, \mathrm{p}<0,00001$ (Figura 2).

\section{Discussão}

Os estudos incluídos eram em sua maioria transversais $^{(21-24)}$ impossibilitando estabelecer relação de causa e efeito. Quanto ao ano de publicação, observou-se que a maioria dos estudos incluídos foram publicados nos últimos cinco anos, ${ }^{(23-26)}$ o que denota ser uma temática de interesse crescente na área da saúde da pessoa idosa. Em uma metanálise evidenciada na literatura ${ }^{(27,28)}$ observou-se que o medo de 
cair e as quedas são importantes problemas de saúde pública que atingem a população idosa, tanto nos países desenvolvidos e em desenvolvimento, o que também foi evidenciado nos estudos desta revisão, estes fenômenos atingem tanto os países em desenvolvimento $^{(23-25)}$ tanto os desenvolvidos. ${ }^{(20-22,26)}$

A diferença encontrada nos estudos quanto à faixa etária para classificar os indivíduos como idosos, em que alguns consideram idosos os indivíduos com 60 anos ou mais ${ }^{(20,22,24)}$ outros $^{(21,23,25,26)} \mathrm{com}$ idade igual ou maior que 65 anos, deve-se ao fato de quem em países em desenvolvimento, de acordo com a Organização Mundial de Saúde serem identificados como idosos a partir de 60 anos, e em países desenvolvidos como Estados Unidos e Canadá, a partir de 65 anos. $\mathrm{O}$ estudo que avaliou os idosos como idade maior ou igual a 72 anos considerou que esta é uma população crescente e que precisa ser estudada. ${ }^{(29)}$

Esta revisão incluiu sete estudos, no entanto, apenas cinco foram incluídos na metanálise. ${ }^{(20-22,25,26)} \mathrm{A}$ justificativa da exclusão de dois estudos ${ }^{(23,24)}$ deu-se afim de diminuir o risco de viés visto que, os mesmos utilizaram diferentes ferramentas de avaliação do medo de cair, interferindo assim na análise dos estudos. Desta maneira, mantiveram-se estudos ${ }^{(20-22,25,26)}$ que utilizaram as seguintes ferramentas de avaliação: uma única pergunta com respostas dicotômicas (sim/ não) e escala analógica (intensidade e frequência).

Quatro estudos ${ }^{(21,22,25,26)}$ apresentaram uma razão de chance de queda, ou seja, um OR superior a 1 no grupo de idosos que responderam que têm medo de cair e possuíam história pregressa de queda, evidenciando assim o medo como um fator de risco para quedas. No entanto, a presença de apenas um único estudo ${ }^{(20)}$ apresentou um OR de 0,42, demonstrando que o medo de cair foi um fator protetor para queda.

No que concerne à heterogeneidade, observouse que foi superior a $50 \%$ em todas as análises realizadas, o que pode ser explicado pelo desenho das publicaçóes incluídas e grande discrepância entre o número e a idade dos pacientes incluídos em cada uma. Uma amostra de tamanho pequeno pode levar a estimativas menos precisas entre a exposição e o desfecho. Além do que, a heterogeneidade pode ele- var-se quando as características dos pacientes, como idade e condiçóes clínicas não serem similares, bem como quando apresentarem diferenças no delineamento e apresentação estatística dos resultados. ${ }^{(12)}$

O Relatório Global da Organização Mundial de Saúde sobre prevenção de Quedas na Velhice, corrobora com o resultado do presente estudo destacando que o medo de cair aumenta o risco de queda entre os idoso que já caíram anteriormente, desencadeando declínio da capacidade funcional (física, mental e sócia) e de gerenciar e evitar outras quedas. ${ }^{(30)}$

Em relação ao instrumento utilizado para avaliar o medo de cair, observou-se que a maioria dos estudos incluídos investigaram o medo de cair por meio de uma pergunta simples, direta com escala analógica, e outros avaliaram por meio das Escala CFES-I e Short FES-I. ${ }^{(23,24)}$

A literatura indica que a utilização de uma única pergunta inicialmente tem vantagens, por ser direta e fácil de gerar estimativas de prevalência de medo de cair na população de idosos. ${ }^{(31)}$ Porém alguns autores ${ }^{(31,32)}$ consideram que esta pergunta apresenta capacidade limitada para detectar a variabilidade de graus de medo, pois pode expressar um estado generalizado de medo que náo reflete diretamente o medo de cair, e dificulta a comparação com outras escalas. Neste sentido, alguns autores ${ }^{(33,34)}$ ampliaram as opçóes de respostas para esta pergunta para refletir melhor o grau de medo de cair como "não ter medo", "medo", "um pouco de medo", "muito medo". Ressalta-se que, além de ser utilizada em diversos estudos, esta pergunta também é adotada pelo Ministério da Saúde do Brasil na Caderneta de Saúde da Pessoa Idosa, merecendo, portanto, ser repensada esta forma de avaliação do medo de cair nesta população. ${ }^{(35,36)}$

A escala FES-I, por sua vez, é fundamentada pela Teoria Cognitiva Social, que apresenta excelente propriedade psicométrica, com consistência interna de 96\%. ${ }^{(37)}$ Essa escala é amplamente utilizada e já foi validada em diversos países, como Brasil, ${ }^{(38)}$ Portugal, ${ }^{(39)}$ Arábia Saudita, ${ }^{(40)}$ Turquia ${ }^{(41)}$ e Espanha. ${ }^{(42)}$ Estes autores apontam que instrumentos que medem a autoeficácia de queda são usados para medir o medo de cair e vice-versa, além do que, existem instrumentos que medem dois constructos distintos (autoeficácia de queda e medo de queda), 
como sendo um único constructo (medo de cair), desta forma, ainda não está clara qual é a melhor escala para medir o medo de cair. ${ }^{(27,33,34)}$

Tendo sido identificado como um fator de risco para quedas, o medo de cair não se encontra nos fatores de risco do Diagnóstico de Enfermagem risco de quedas, conforme evidenciado na Classificação Internacional de Diagnósticos de Enfermagem da NANDA-I, desta maneira, a sua inclusão poderá contribuir para o refinamento deste diagnóstico. Sugere-se a realização de estudos teóricos de análise de conceito do medo de cair, pois eles podem sustentar a construção de escalas que possibilitarão avaliar esta condição nesta população, além de estudos com metodologias mais robustas para estabelecer a relação causa e efeito. Recomenda-se que esta temática seja abordada no processo de formação dos profissionais de saúde, nos serviços de atenção à saúde, públicos ou privados. Os enfermeiros, bem como a equipe multiprofissional que atuam diretamente com esta população, devem investigar o risco de quedas para propor possíveis medidas preventivas.

\section{Conclusão}

O presente estudo aponta que o medo de cair é um fator de risco para queda em idosos que vivem na comunidade e que possuem história pregressa de queda, tornando-se emergente a inclusão deste aspecto na assistência dos enfermeiros e equipe multiprofissional a esta população. Embora tenham sido identificados pouco estudos que estabeleçam relação de causa e efeito, foi possível estabelecer uma razão de chance de queda de 12 a 15 vezes para o grupo de idosos com medo de cair, quando comparado ao grupo de idosos sem medo de cair que vivem na comunidade. Acredita-se que novas pesquisas com métodos robustos de avaliação do medo de cair devem ser desenvolvidos em idosos que já tiveram quedas ou não.

\section{Agradecimentos}

Ao Conselho Nacional de Desenvolvimento Científico e Tecnológico (CNPq) pela bolsa de pro- dutividade da Profa Dra Alba Lucia Bottura Leite de Barros - Coordenação de Aperfeiçoamento de Pessoal de Nível Superior (CAPES) bolsa de Doutorado.

\section{Referências}

1. World Health Organization (WHO). World report on ageing and health [Internet]. Luxembourg: WHO; 2015. [cited 2018 Mai 20]. Available from: http://apps.who.int/iris/ bitstream/10665/186463/1/9789240694811_eng.pdf

2. Vitorino LM, Teixeira CA, Vilas Boas EL, Pereira RL, Santos NO, Rozendo CA. Fear of falling in older adults living at home: associated factors. Rev Esc Enferm USP. 2017; 51:e03215.

3. Oh E, Hong GRS, Lee S, Han S. Fear of falling and its predictors among community-living older adults in Korea. Aging Ment Health. 2017; 21:369-78.

4. Murphy J, Issacs B. The post-fall syndrome: A study of 36 elderly patients. Gerontology.1982; 28(4):265-70.

5. Hadjistavropoulos T, Delbaere K, Fitzgerald TD. Reconceptualizing the role of fear of falling and balance confidence in fall risk. J Aging Health. $2011 ; 23: 3-23$

6. Brasil. Ministério da Saúde, Secretaria de Atenção à Saúde. Departamento de Ações Programáticas Estratégicas. Manual para Utilização da Caderneta de Saúde da Pessoa Idosa. Brasília, DF: Ministério da Saúde; 2016. 94p.

7. Tinetti ME, Richman D, Powell L. Falls efficacy as a measure of fear of falling. J Gerontol. 1990;45(6):P239-43.

8. Kempen Gl, Yardley L, Van Haastregt JC, Zijlstra GA, Beyer N, Hauer K, Todd C. The Short FES-I: a shortened version of the falls efficacy scale-international to assess fear of falling. Age Ageing. 2008; 37(1):45-50.

9. Herdman TH, Kamitsuru S. NANDA. Diagnóstico de enfermagem da NANDA: definições e classificação 2018-2020. 10a ed. Porto Alegre: Artmed; 2018.

10. Park Jl, Yang JC, Chung S. Risk Factors Associated with the Fear of Falling in Community-Living Elderly People in Korea: Role of Psychological Factors. Psychiatry Investig. 2017;14(6):894-99.

11. Chang HT, Chen HC, Chou P.Fear of falling and mortality among communitydwelling older adults in the Shih-Pai study in Taiwan: A longitudinal followup study. Geriatr Gerontol Int. 2017;17(11):2216-23.

12. Brasil. Ministério da Saúde, Secretaria de Ciência, Tecnologia e Insumos Estratégicos. Departamento de Ciência e Tecnologia. Diretrizes Metodológicas: elaboração de revisão sistemáticas e metanálise de estudos observacionais comparativos sobre fatores de risco e prognósticos. Brasília, DF: Ministério da Saúde; 2014b.

13. Howland J, Peterson EW, Levin WC, Fried L, Pordon D, Bak S. Fear of falling among the community dwelling elderly. J Aging Health. 1993;5:229-43.

14. King MD, Tinetti ME. Falls in community-dwelling older persons. J Am Geriatr Soc. 1995; 43(10):1146-54.

15. Friedman SM, Munoz B, West SK, et al. Falls and fear of falling: which comes first? A longitudinal prediction model suggests strategies for primary and secondary prevention. J Am Geriatr Soc. 2002;50(8):1329-35. 
16. Arfken CL, Lach HW, Birge SJ, J P Miller. The prevalence and correlates of fear of falling in elderly persons living in the community. Am J Public Health.1994; 84:565-70.

17. Chu LW, Chi I, Chiu AY. Incidence and predictors of falls in the Chinese elderly. Ann Acad Med Singapore. 2005;34(1):60-72. Erratum in: Ann Acad Med Singapore. 2005; 34(7):469.

18. Coll-Planas L, Kron M, Sander S, Rissmann U, Becker C, Nikolaus T. Accidental falls among community-dwelling older adults: improving the identification process of persons at risk by nursing staff. Z Gerontol Geriatr. 2006; 39(4):277-82.

19. Chou K-L, Chi I. The temporal relationship between falls and fearof-falling among Chinese older primary-care patients in Hong Kong. Ageing Soc. 2007;27(2):181-93.

20. Lach HW. Incidence and risk factors for developing fear of falling in older adults. Public Health Nurs. 2005; 22(1):45-52.

21. Filiatrault J, Desrosiers J. Coping strategies used by seniors going through the normal aging process: does fear of falling matter? Gerontology. 2011; 57:228-36.

22. Kim S, So WY. Prevalence and correlates of fear of falling in Korean community-dwelling elderly subjects. Exp Gerontol. 2013; 48(11):1323-8.

23. Liu JY. Fear of falling in robust community-dwelling older people: results of a cross-sectional study. J Clin Nurs. 2014; 24(3-4):393-05.

24. Mane AB, Sanjana T, Patil PR, Sriniwas T. Prevalence and correlates of fear of falling among elderly population in urban area of Karnataka, India. J Midlife Health.2014; 5(3):150-5.

25. Dierking L, Markides K, Snih SAL, Kristen Peek M. Fear of Falling Among Older Mexican Americans: A Longitudinal Study of Incidence and Predictive Factors. J Am Geriatr Soc. 2016; 64(12):2560-5.

26. Lavedán $A$, Viladrosa $M$, Jürschik $P$, et al. Fear of falling in communitydwelling older adults: A cause of falls, a consequence, or both? Journal Pone. 2018; Mar 29;13(3):1-14.

27. Scheffer AC, Schuurmans MJ, van Dijk N, van der Hooft T, de Rooi SE. Fear of falling: measure strategy, prevalence, risk factor and consequences among older persons. Age Ageing.2008; 37:1924.

28. World Health Organization (WHO). Active ageing: A Policy Framework. Aging Male [Internet]. 2002 [cited 2017 Mai 15];5(1):1-37. Genève: WHO. Available from: http://www.who.int/ageing/publications/active_ ageing/en/

29. Centers for Disease Control and Prevention. Falls-Older Adults. Home and Recreational Safety. Atlanta, GA: Centers for Disease Control and Prevention; 2012
30. World Health Organization (WHO). Global report on falls prevention in older age. France: WHO Library Cataloguing-in-Publication Data; 2007 [Internet]. 2007 [cited 2017 Mai 15]. Available from: http://www.who. int/ageing/publications/Falls_ prevention7March.pdf

31. Jung D. Fear of Falling in Older Adults: Comprehensive review. Asian Nurs Res. 2008; 2(4):214-22.

32. Zijlstra GA, van Haastregt JC, Van Eijk JT, Van Rossum E, Stalenhoef PA, Kempen Gl. Prevalence and correlates of fear of falling, and associated avoidance of activity in the general population of community-living older people. Age Ageing. 2007; 36(3):304-9.

33. Jørstad EC, Hauer K, Becker C, Lamb SE; ProFaNE Group. Measuring the psychological outcomes of falling: a systematic review. J Am Geriatr Soc. 2005;53(3):501-10

34. McAuley EM, Mihalko SL, Rosengren K. Self-efficacy and balance correlates of fear of falling in the elderly. J Aging Phys Act. 1997; 5:329-40.

35. Brasil. Ministério da Saúde, Secretaria de Atenção à Saúde. Departamento de Ações Programáticas Estratégicas. Manual para Utilização da Caderneta de Saúde da Pessoa Idosa. Brasília: Ministério da Saúde; 2016. 94p.

36. Choi K, Jeon GS, Cho Sl. Prospective study on the impact of fear of falling on functional decline among community dwelling elderly women. Int J Environ Res Public Health. 2017;14(5). pii: E469.

37. Yardley L, Beyer N, Hauer K, et al. Development and initial validation of the Falls Efficacy Scale-International (FES-I). Age Ageing. 2005; 34(6):614-9.

38. Camargos FF, Dias RC, Dias JM, Freire MT. Adaptação transcultural e avaliação das propriedades psicométricas da Falls Efficacy Scale - International em idosos Brasileiros (FES-I-BRASIL). Rev Bras Fisioter. 2010;14(3):237-43.

39. Marques VC, Sousa LM, Sousa LM, Berenguer SM. Validation of the falls efficacy scale - international in a sample of Portuguese elderly. Rev Bras Enferm. 2018;71(Suppl 2):747-54.

40. Ahmad H. Algadir, Murad Al-Momani, Gregory F. Marchetti, Susan L. Whitney. Cross-cultural adaptation and measurement properties of the Arabic version of the Fall Efficacy Scale International. Neurociências (Riade). 2015; 20 (3): 230-5.

41. Kovács E, Rozs F, Petridisz A, Erdős R, Majercsik E. Cross-cultural validation of the Falls Efficacy Scale-International to assess concerns about falls among Hungarian community-living older people. Disabil Rehabil. 2018;40(25):3070-5

42. Lomas-Vega R, Hita-Contreras F, Mendoza N, Martínez-Amat A. Crosscultural adaptation and validation of the Falls Efficacy Scale International in Spanish postmenopausal women. Menopause. 2012; 19(8):904-8. 